\title{
RESEARCH ON NOISE REDUCTION AND ENHANCEMENT OF WELD IMAGE
}

\author{
Xiang-Song Zhang ${ }^{1}$, Wei-Xin $\mathrm{Gao}^{1}$ and Shi-Ling Zhu ${ }^{2}$ \\ ${ }^{1}$ College of Electronic Engineering, Xi'an Shiyou University, Xi'an, China \\ ${ }^{2}$ Communication and Information Engineering, Xi' an University of Post and \\ Telecommunications, Xi'an, China
}

\begin{abstract}
In order to eliminate the salt pepper and Gaussian mixed noise in X-ray weld image, the extreme value characteristics of salt and pepper noise are used to separate the mixed noise, and the non local mean filtering algorithm is used to denoise it. Because the smoothness of the exponential weighted kernel function is too large, it is easy to cause the image details fuzzy, so the cosine coefficient based on the function is adopted. An improved non local mean image denoising algorithm is designed by using weighted Gaussian kernel function. The experimental results show that the new algorithm reduces the noise and retains the details of the original image, and the peak signal-to-noise ratio is increased by $1.5 \mathrm{~dB}$. An adaptive salt and pepper noise elimination algorithm is proposed, which can automatically adjust the filtering window to identify the noise probability. Firstly, the median filter is applied to the image, and the filtering results are compared with the pre filtering results to get the noise points. Then the weighted average of the middle three groups of data under each filtering window is used to estimate the image noise probability. Before filtering, the obvious noise points are removed by threshold method, and then the central pixel is estimated by the reciprocal square of the distance from the center pixel of the window. Finally, according to Takagi Sugeno (T-S) fuzzy rules, the output estimates of different models are fused by using noise probability. Experimental results show that the algorithm has the ability of automatic noise estimation and adaptive window adjustment. After filtering, the standard mean square deviation can be reduced by more than $20 \%$, and the speed can be increased more than twice. In the enhancement part, a nonlinear image enhancement method is proposed, which can adjust the parameters adaptively and enhance the weld area automatically instead of the background area. The enhancement effect achieves the best personal visual effect. Compared with the traditional method, the enhancement effect is better and more in line with the needs of industrial field.
\end{abstract}

\section{KEYWORDS}

X-ray image, Mixed noise, Noise separation, noise reduction, image enhancement.

\section{INTRODUCTION}

Due to the influence of equipment and acquisition environment in X-ray welding image acquisition, welding images are limited by low contrast and large noise, which will interfere with the segmentation and recognition of welding image defects. With the development of image processing technology, image denoising and contrast enhancement methods have emerged in large numbers, and some achievements have been achieved. The weld images obtained by X-ray generally are characteristic of fuzzy defect edges and various image noise [1]. To detect internal defects more accurately, researchers should reduce noise and enhance the weld images. There are many research findings in these fields. For instance, an improved Gabor filter image contrast denoising algorithm was proposed, which can reduce the noise and retain the image details and David C. Wyld et al. (Eds): SPPR, NECO, GridCom, ICCSEA, SCAI, UBIC, SEMIT, MLDS - 2020 pp. 13-26, 2020. CS \& IT - CSCP 2020 
defect areas [2]. A local image enhancement method was proposed by determining the local pixel nonuniformity factor, and the combination of histogram equalization and contrast limitation can adaptively improve the defect detection accuracy [3]. However, due to the large noise of weld images, the contrast is low and the difference between the image background and the target gray is not obvious. If the nonuniformity factor is not set accurately, the background and the target will be enhanced, which interferes with the realization of the enhancement effect. A new anisotropic diffusion imaging enhancement method can smooth, maintain edges and sharpen at the same time [4]. We compared various denoising methods based on anisotropic diffusion, and evaluated their performances with mean square error (MSE) and peak signal-to-noise ratio (PSNR), which underlay further image segmentation and feature extraction experiments on noisy weld X-ray images [5]. Firstly, wavelet denoising was used to remove the random high-frequency noise generated in the detection of scattering radiation, then Shape from Shading(SFS) method was used to distinguish the moire optical characteristics of low- and high-gradient defect areas, and finally, Harris corner detection(HCD) method was adopted to detect and highlight the types of residual corners related to different welding defects [6]. Because the effect of wavelet denoising on Gaussian noise is obvious and the noise in a weld image is Gaussian and salt-pepper mixed noise, the effect of wavelet de-noising alone is not ideal. A gas tungsten arc weld (GTAW) deep learning enhancement method based on multi-source remote sensing image was proposed [7]. Two integrated methods including generative antagonism network and classic convolution neural network were designed, which combined multiple neural networks to improve the modeling performance for the unobserved data in different experimental environments. An enhancement method based on the scale variable stochastic resonance model and a stochastic resonance image enhancement method based on the genetic optimization algorithm were proposed, which made full use of the parallel optimization of the genetic algorithm and the weak signal enhancement of stochastic resonance and overcame the shortcomings of traditional methods [8]. A series of improvement measures based on the similarity between local blocks of the image were put forward [9]. Local binary pattern (LBP) texture feature and edge structure tensor were used to improve the comparison of similar blocks and the effect of noise reduction. In a two-step noise suppression based method for low-light-level image perception enhancement, firstly the noise level function was used for contrast enhancement of noise perception, and then the just-noticeable-distortion (JND) model was used to reduce the noise while keeping the details, estimate noise visibility according to the intensity change of brightness and extract the details through contrast masking and visual regularity [10]. With an improved nonlocal mean filtering model, the rotation angle between image blocks was calculated by using the main direction of corner points, and the similarity between blocks was computed more accurately [11]. However, the noise reduction result was not ideal for weld images, because of the overall low contrast of weld images and the indistinguishable similarity between image blocks [11]. When histogram equalization algorithm was combined with high-frequency lifting filter, the image information entropy and contrast after processing were higher and the processing time was shorter, which can meet the real-time requirements of image processing [12]. In an adaptive noise reduction method of image sensor, the image was decomposed by curve transform and the variational technique was used to reduce noise and improve PSNR [13]. In a laser 3D image enhancement system based on virtual reality, point cloud data were used to build a 3D image model that conformed to normal distribution [14]. According to the distribution of its point image elements, the image was enhanced effectively by the 3D image transformation enhancement system. A low-illuminance nonlinear laser image edge adaptive enhancement device based on radial hill was adopted [15]. Moreover, an nsmission estimation method based on the improved dual region filter and the guide filter was proposed to reduce the complexity of image defogging algorithms and solve the white halo of image edges, and was verified experimentally to be effective and efficient [16].In reference [17], an adaptive dynamic weighted median filtering algorithm is proposed, which achieves good filtering effect. In reference [18], a block median filtering algorithm based on probability decision-making is proposed, which improves the traditional median filtering, and the 
effect is remarkable.[19] An iterative nonlocal mean filtering algorithm is proposed to remove impulse noise.[20]In this paper, a convolutional neural network (CNN) model algorithm based on deep learning is proposed. The algorithm is superior to sparse representation and block based method in accuracy and robustness.

Based on the above analyses and in view of the Gaussian and salt-and-pepper mixed noise in weld images, firstly noise separation was carried out, then an improved nonlocal mean filter was proposed to reduce the Gaussian noise and finally, an adaptive Takagi-Sugeno (T-S) fuzzy fusion filter algorithm which can identify the probability of salt and pepper noise was proposed in combination with the median filter. To improve the image contrast, we proposed an adaptive parameter adjustment enhancement algorithm without human intervention. The enhancement effect of the algorithm can reach the best vision of human eyes. Finally, experiments show that the proposed algorithm has obvious advantages in noise reduction and enhancement.

\section{IMAGE NOISE REDUCTION}

The noise of an X-ray weld image is mainly the photoelectron noise caused by the photoelectron conversion of the image sensor during shooting, and the electronic noise caused by the random thermal movement of electrons. Based on mathematical analysis, the noise can be mainly divided into Gaussian noise and salt-and- pepper noise. The noise in an actual image is a mixture of the two. The model of the mixed noise can be expressed as follows:

$$
f(i, j)=f_{y}+z_{g}+z_{s}
$$

where $f(i, j)$ and $f_{y}$ represent the image with mixed noise and the original image respectively; $z_{g}$ and $z_{s}$ represent Gaussian noise and salt-and-pepper noise respectively.

\subsection{Noise Model}

As for Gaussian noise, its probability density is described by Gaussian normal distribution. A larger mean value of Gaussian noise means a whiter picture; the larger variance indicates the image is at lower resolution and is more fuzzy. Such noise in a digital image is mainly caused by uneven illumination or high temperature.

$$
p(z)=\frac{1}{\sqrt{2 \pi} \sigma} e^{-(z-\mu)^{2} / 2 \sigma^{2}}
$$

where $p(z)$ represents the probability of the gray value $z, \mu$ and $\sigma^{2}$ are the mean and variance.

Salt and pepper noise refers to two kinds of noise: salt noise and pepper noise. The white noise is salt noise and has a high gray value (255), and the black noise is pepper noise and has a low gray value (0). $a$ and $b$ represent the limit gray values in the image.

$$
p(z)= \begin{cases}p_{a} & z=a \\ p_{b} & z=b \\ 0 & \text { else }\end{cases}
$$




\subsection{Mixed Noise Separation}

The gray values of salt and pepper noise points are characteristic of extreme values, and are generally the positive (negative) maxima or minima of gray value in the region where they are located. However, these positive (negative) maxima or minima may not be noise pixels, because there are also positive (negative) maxima or minima in the undisturbed image gray smooth area or the area with obvious edge. Therefore, it is necessary to further judge whether the extreme point is a salt and pepper noise point. Let an $m \times n$ window with $x(i, j)$ be the center. If $x(i, j)=f(i, j)_{\text {max }}$, count the pixels $x(i, j) \neq f(i, j)_{\text {max }}$ in the window, where there are $n_{1}$ in all; if $x(i, j)=f(i, j)_{\min }$, count the pixels $x(i, j) \neq f(i, j)_{\min }$ in the window, where there are $n_{2}$ in all. When $n_{1}>t$ or $n_{2}>t$ ( $t$ is the threshold value of salt and pepper noise and generally varies between 12 and 16), the pixel point is judged as a salt and pepper noise point; when $n_{1}<t$ or $n_{2}<t$, it is not judged as a salt and pepper noise point.

\subsection{Gauss Noise Filtering}

\subsubsection{Nonlocal Mean Filtering}

The basic idea of original nonlocal mean (onlm) filtering is to use a large amount of redundant information in the image to calculate the weighted average value of gray values of all pixels in the same neighborhood according to the weighted coefficient. The core problem is to determine the weighted kernel function by the weighted Euclidean distance between this function and the image. The exponential kernel function (EKF) used for weighting makes the image details too smooth and fuzzy. Therefore, on the basis of previous works, we discuss the establishment of the weighted kernel function and propose a new cosine Gaussian kernel function (CGKF) combining the Gaussian kernel function (GKF) and the cosine kernel function (CKF). The noise signal is assumed to be image-independent additive Gaussian white noise, and can be modelled as follows:

$$
Z(i)=X(i)+N(i)
$$

where $X(i)$ is the original image without noise pollution; $N(i)$ is the Gaussian white noise with mean value of 0 and variance of $\sigma^{2} ; Z(i)$ is the noise-contaminated image. Let $z=\{z(i) \mid i \in I\}$ be a pair of discrete noisy images, where $I$ is the image domain. For any pixel $i$ in the image, the onlm filter uses the weighted average of all pixel gray values in the image to estimate the gray value of the point:

$$
N L[z](i)=\sum_{j \in I} w(i, j) z(i)
$$

where the weight $z(i)$ depends on the similarity between pixels $i$ and $j$, and satisfies $0<z(i)<1$ and $\sum_{j} z(i, j)=1$. The similarity between pixels $i$ and $j$ is determined by the similarity between gray value matrices $H_{i}$ and $H_{j}$, where $H_{i}$ represents a known-size square neighborhood centered on pixel $i$.The similarity between the gray value matrices of each neighborhood is measured by their Gaussian weighted Euclidean distance $d(i, j)$ :

$$
d(i, j)=\left\|H_{i}-H_{j}\right\|_{2, \alpha}^{2}
$$


where $\alpha$ is the standard deviation weighted by Gauss and $\alpha>0$; Function \|\|$_{2}$ is $L^{2}$ norm, so a higher neighborhood similarity indicates a smaller distance. Weight $w(i, j)$ is defined as:

$$
w(i, j)=\frac{1}{C(i)} f_{k}(d(i, j))
$$

where $C(i)=\sum_{j} f_{k}(d(i, j))$ is the normalized parameter. The core problem of nonlocal mean (nlm) filtering given in Eq. (6) is the kernel function $f_{k}($.$) in Eq. (8) and it plays an important role in the$ denoising performance of the algorithm. The nlm method adopts EKF, which is defined as:

$$
f_{k}(d(i, j))=\exp \left(-\frac{d(i, j)}{h^{2}}\right)
$$

where $h$ is the attenuation factor, which controls the attenuation speed of the exponential function and affects the filtering degree and denoising performance of the algorithm.

\subsubsection{Improved NIm Filtering}

The core problem of nlm filtering is to determine the weighted kernel function. The core idea of weighting is to give more weight to the neighborhood with higher similarity, and less weight to the neighborhood with lower similarity. This is because the neighborhood with low similarity or dissimilarity will increase the computation of the algorithm and affect its denoising effect. Under certain conditions, the ideal kernel function should output larger weights when the distance between pixels is small, and the output will decrease rapidly to 0 as the distance is enlarged. The weighted kernel function plays an important role in denoising performance, including the cosine type and the Gauss type. Specifically, CKF is defined as:

$$
f_{k}(d(i, j))=\left\{\begin{array}{cc}
\cos (\pi d(i, j) / 2 h) & d(i, j) \leq h \\
0 & d(i, j)>h
\end{array}\right.
$$

and GKF is defined as:

$$
f_{k}(d(i, j))=\exp \left(-\frac{d^{2}(i, j)}{h^{2}}\right) \quad d(i, j) \leq h
$$

When the noise intensity is weak, the denoising performance is better than that of $\mathrm{nlm}$, but the over weighting of CKF and the insufficient weighting of GKF lead to the decrease of denoising performance when the signal intensity increases. Based on the comparative analysis of EKF, CKF and GKF, a new cosine Gaussian kernel function (CGKF) was proposed:

$$
f_{k}(d(i, j))=\left\{\begin{array}{cc}
\exp \left(-\frac{d^{2}(i, j)}{h_{1}^{2}}\right) \cos \left(\pi d(i, j) / 2 h_{2}\right) & d(i, j) \leq h_{2} \\
0 & d(i, j)>h_{2}
\end{array}\right.
$$

where $h_{1}$ and $h_{2}$ are filter parameters. CGKF adds a cosine coefficient on basis of GKF, so the improved algorithm can perform denoising better at different noise levels. In comparison of Eq. (8) to Eq. (11), the response curve is shown in Figure 1. 


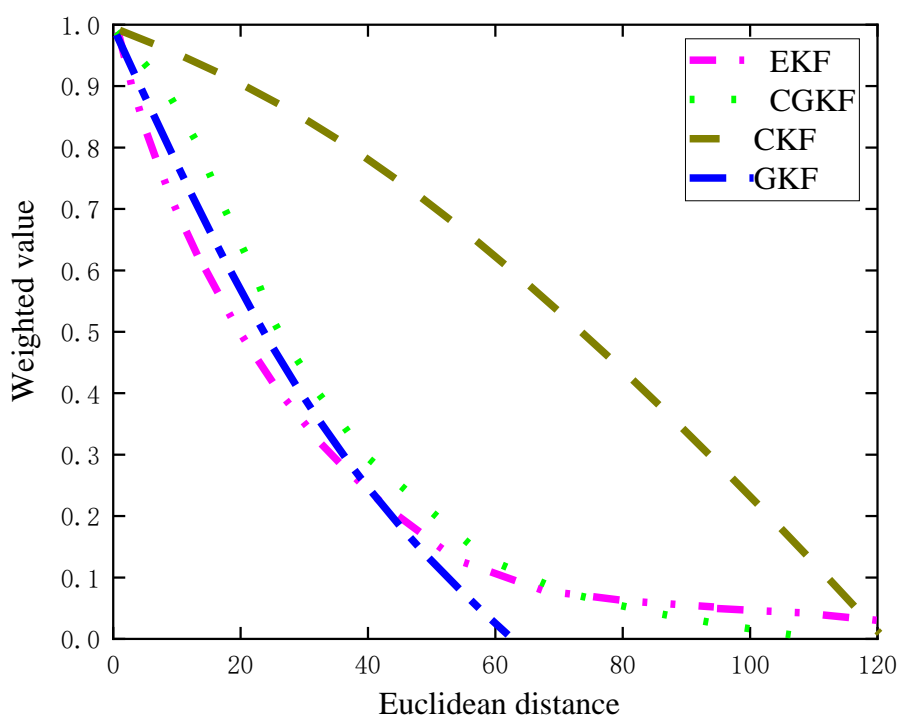

Figure 1. Response curve of weighted kernel function

Compared with the EKF of onlm, the response curve of GKF is flatter when the distance between pixels is large, and drops rapidly as the distance increases, and the response curve of CKF is relatively flat in the whole region (Fig. 1). The improved CGKF synthesizes their advantages and has greater weighting when the distance is small, and the weight decreases at the enlarged distance, avoiding over-weighting or under-weighting. Hence, we can make full use of the highsimilarity neighborhood to filter out noise, and effectively reduce the interference of lowsimilarity and dissimilar neighborhood. Experimental results show that the denoising performance is better than that of the onlm algorithm.

\subsection{Salt and pepper noise filtering}

For salt and pepper noise, the median filter is obviously effective on it, but has a fixed window. Firstly, a small window such as a $3 \times 3$ template can effectively retain image details, but its noise reduction effect is not good when the noise density increases. Secondly, a large window such as a $5 \times 5$ template can effectively reduce salt and pepper noise, but loses the image details. Moreover, median filtering cannot estimate the probability of salt and pepper noise. Therefore, we propose an adaptive salt and pepper noise elimination algorithm that can automatically adjust the filtering window to identify the noise probability. This algorithm is described in detail below.

\subsubsection{Noise Probability Estimation}

The salt and pepper noise probability is estimated as follows:

Step1 After the image is partitioned, select any point in each area, and take $S_{1}, S_{2} \ldots S_{n}, n$ modules centered on this point;

Step2 Use the adaptive median filtering window of $3 \times 3,5 \times 5,7 \times 7$ to filter $n$ regions, and $3 n 3 \times 3$ rectangular regions, and obtain $S_{11}^{\prime}, S_{12}^{\prime} \ldots S_{1 n}^{\prime}, S_{21}^{\prime}, S_{22}^{\prime} \ldots S_{2 n}^{\prime} S_{31}^{\prime}, S_{32}^{\prime} \ldots S_{3 n}^{\prime}$;

Step3 In different filtering windows, subtract each point in the filtered area $S_{11}^{\prime}, S_{12}^{\prime} \ldots S_{1 n}^{\prime}, S_{21}^{\prime}, S_{22}^{\prime} \ldots S_{2 n}^{\prime}, S_{31}^{\prime}, S_{32}^{\prime} \ldots S_{3 n}^{\prime}$ from the gray level of each point in the previous 
area $S_{1}, S_{2} \ldots S_{n}$. After the absolute value of the subtracted result is taken, count the points over a certain positive number $u_{11}, u_{12} \ldots u_{1 n} ; u_{21}, u_{22} \ldots u_{2 n} ; u_{31}, u_{32} \ldots u_{3 n}$;

Step4 Regroup the number of pixels $u_{i j}(i=1,2 \ldots n, j=1,2 \ldots n)$ in any small area, and take the average value of the middle $m$ pixels in the small area, i.e. $a_{1} 、 a_{2} 、 a_{3}$.The weighted average of $a_{1} 、 a_{2} 、 a_{3}$ is $9 m$ higher than the noise probability.

\subsubsection{T-S fuzzy Model Fusion Filtering}

To facilitate the following discussion, let the image size be $M \times N$, any pixel point in the image be $f(i, j)$, and the filtered pixel be $f^{\prime}(i, j)$, where $i=1,2,3 \ldots M, j=1,2,3 \ldots N$. The defect of the median filter is that it can rank all pixels without the ability of noise point recognition and noise rate estimation. Herein, the variable scale sliding window is used to flexibly adopt windows of different sizes for different noise probabilities. It can reduce the computation amount and the blur caused by relatively large windows when the noise probability is low, and can improve the filtering accuracy when the noise probability is high. Figure 2 shows four window modes, which will be combined into four filtering modes, where (a) to (d) are $\mathrm{Model}_{1}, \mathrm{Model}_{2}, \mathrm{Model}_{3}$ and $\mathrm{Model}_{4}$, respectively and "Star" is the pixel participating in filtering.

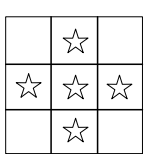

(a)

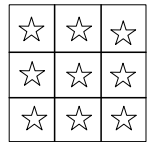

(b)

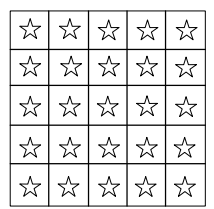

(c)

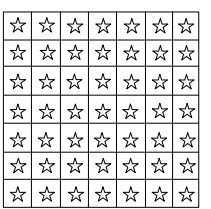

(d)

Figure 2. Maximum window model

(1) $F M_{1}$ (abbreviation of $F_{i l t e r} M_{\text {odel }}$ ), window, Model $l_{1}$ is sliding filter window, and window Model $_{2}$ as the backup is the largest window;

(2) $F M_{2}: F M_{2}$ is the main window and $F M_{3}$ is the backup;

(3) $F M_{3}: F M_{3}$ is the main window and $F M_{4}$ is the backup;

(4) $F M_{4}: \quad F M_{4}$ is the main window.

After determination of noise probability, the fusion filtering results of different noise probability values and fuzzy correspondence under T-S model of filtering model are given in Figure. 3.

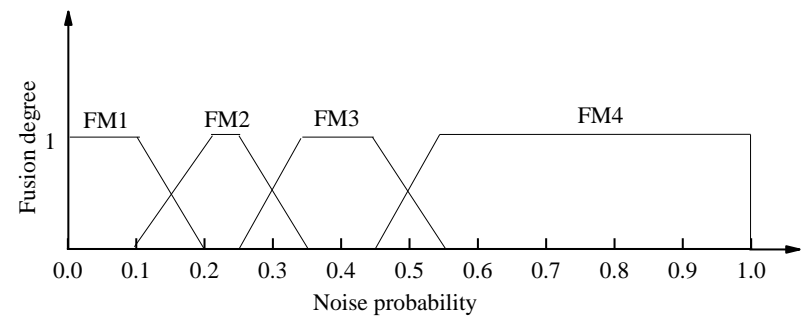

Figure 3. Corresponding fuzzy relation between noise probability and filtering mode 


\subsubsection{Filtering Algorithm}

Based on the above analysis, the steps of the adaptive salt and pepper noise elimination algorithm proposed here are as follows:

Step1 Noise probability calculation;

Step2 Model fusion: according to the noise probability, the filtering algorithm under the T-S model is used. If the probability of salt and pepper noise is estimated to be $0.4, \mathrm{FM}_{3}$ is used; if the probability is $0.5, F_{3}$ and $F M_{4}$ are filtered at the same time. Finally, the results of $F M_{3}$ and $\mathrm{FM}_{4}$ are fused and outputted according to the T-S fuzzy relationship.

Filtering under the known model: firstly, the pixel $v(i, j)$ is detected. If the pixel is not noisepolluted, the result according to Eq. (12) is outputted and then the next pixel $v(i, j+1)$ is filtered; otherwise, the pixels of the filtering window in the filtering mode are detected one by one, and the number of non-noise-polluted pixels $n n$ is counted. If $n n \neq 0$, the output of noise pixel is estimated as per Eq. (13); if $n n=0$, the detection range of the pixel is extended to the range of the standby window and Eq. (14) is adopted:

$$
\begin{array}{ll}
v^{\prime}(i, j)=v(i, j) & \min <v(i, j)<\max \\
v^{\prime}(i, j)=\sum_{m}^{R} \sum_{n}^{R} \omega_{n n} v(m, n) / \sum_{m}^{R} \sum_{n}^{R} \omega_{m n} & v(i, j)<\min \text { 或 } v(i, j)>\max \text { 且 } n n \neq 0 \\
v^{\prime}(i, j)=\operatorname{med}\left(W_{i j}\right) & v(i, j)<\min \text { 或 } v(i, j)>\max \text { 且 } n n=0
\end{array}
$$

where $v(i, j)$ is the center pixel of the current window $W_{i j}(R \times R, R$ is odd $) ; v^{\prime}(i, j)$ is the estimated value after filtering of the current operating pixel; min and max are the noise thresholds; Med is the median value of the pixel, $\omega_{m n}$ is the weighting of the undisturbed pixel, i.e. $1 /\left(x^{4}+y^{4}\right) ; x$ and $y$ are the horizontal and vertical pixel spacing respectively from any pixel point to the center pixel point.

\section{IMAGE ENHANCEMENT}

\subsection{Sin function transformation}

The gray-scale transformation curve of a sin function is characterized by gentle transformation of upper and lower wave heads, and large changes in the middle (Fig. 4). Since the gray level of the weld image with poor contrast concentrates in a certain range and the gray level of the range is stretched by the sin function, the gray level of the background area larger than the average gray value increases and that of the weld area smaller than the average gray value decreases. This feature is very effective in distinguishing the gray values of the background area and the weld area. The transformation method of the sin function is (15):

$$
g(x, y)=127\left\{1+\sin \left[\frac{\pi \cdot f(x, y)}{b-a}-\frac{\pi \cdot(a+b)}{2 \cdot(b-a)}\right]\right\}
$$


where $f(x, y)$ and $g(x, y)$ are the gray values before and after transformation respectively; $a$ and $b$ are the lowest and highest gray values before transformation respectively. The gray ranges of the sin function before and after transformation are the same.

\subsection{Logarithmic transformation}

The expression of logarithmic transformation is:

$$
g(x, y)=a+\frac{\ln [f(x, y)+1]}{b \cdot \ln c}
$$

where $a, b$ and $c$ are parameters for adjusting the position and shape of the curve. Logarithmic transformation can stretch the low-gray area of the image and compress the high-gray area. The transformation curve is shown in Fig. 5.

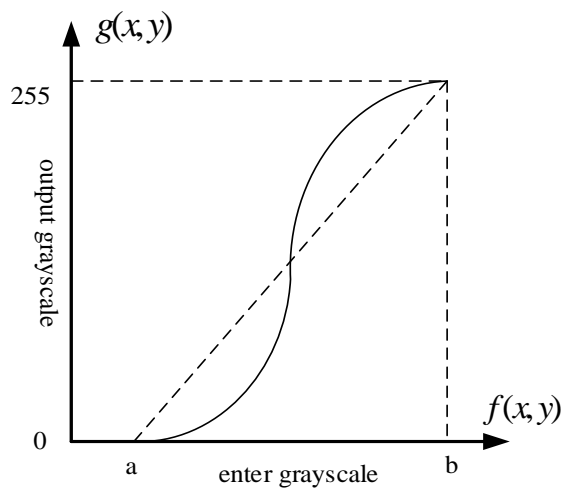

Figure 4. Sin transformation curve

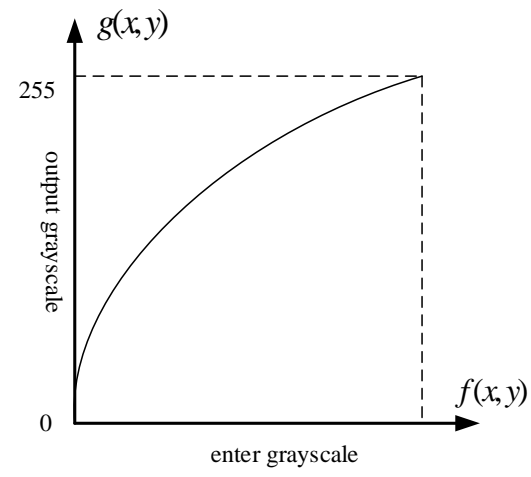

Figure 5. Logarithmic transformation curve

\subsection{XE function transformation}

At present, the image of X-ray pipeline girth weld is fuzzy and low-quality. Neither manual nor automatic detection is conducive to identify the defects in the weld. The existing image enhancement algorithms mostly use histogram dynamic range stretching, which can stretch both the target area and the background area. However, there is still a lack of X-ray image enhancement technology for girth weld region of interest without parameter selection. Herein, a method of industrial X-ray girth weld image enhancement was used. The gray value of a weld area was determined by retrieving the gray value corresponding to the second peak of gray histogram. The gray value of the weld area was enhanced by introducing a specific continuous function, and that of background was suppressed. It can automatically enhance the weld area instead of the background area, without manual intervention in parameter selection, and has strong robustness. To effectively enhance the X-ray image of girth weld to the best value of personal visual perception, we conducted a number of experiments. The success rate of defect detection is improved from about $95 \%$ without enhancement to about $98 \%$ after the enhancement of xe function. The gray-scale enhancement is expressed as follows, $f(\operatorname{gray}(i, j)), g(\operatorname{gray}(i, j))$ is the gray value before and after enhancement.The xe enhancement function is shown in Figure 6 


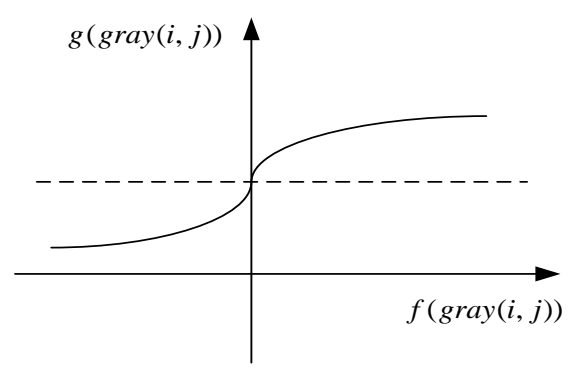

Figure 6. Schematic diagram of xe function enhancement

Because the collected girth weld images are placed horizontally, the xe function enhancement method is designed, as shown in the figure above. It can be seen from the figure that the closer the xe function image is to 0 , the more obvious the change of gray level is.

\section{EXPERIMENTAL ANALYSIS}

\subsection{Peak signal-to-noise ratio(PSNR)comparison after filtering}

Totally 200 weld images were randomly selected from the image library and were filtered by the nlm filter with different kernel functions. After that, 10 filtered images were randomly selected and numbered as 1 to 10 . The PSNR of these 10 images were compared with that of mean filter (meaf), median filter (medf) and algorithm in Ref. 10. To write conveniently, we used different kernel functions to represent these images. For different filters, the PSNRs of the filtered images are shown in Table 1.

Table 1. PSNR of filtered image

\begin{tabular}{llllllll}
$\begin{array}{l}\text { Image } \\
\text { No. }\end{array}$ & $\begin{array}{l}\text { CGKF } \\
\text { psnr }\end{array}$ & $\begin{array}{l}\text { MEAF } \\
\text { psnr }\end{array}$ & $\begin{array}{l}\text { GKF } \\
\text { psnr }\end{array}$ & $\begin{array}{l}\text { EKF } \\
\text { psnr }\end{array}$ & $\begin{array}{l}\text { MEDF } \\
\text { psnr }\end{array}$ & $\begin{array}{l}\text { CKF } \\
\text { psnr }\end{array}$ & $\begin{array}{l}\text { Document } \\
10 \text { psnr }\end{array}$ \\
\hline 1 & 53.4475 & 52.8513 & 50.4475 & 48.8328 & 47.8411 & 51.6415 & 48.4135 \\
2 & 54.7413 & 53.2919 & 50.7413 & 48.0659 & 47.5142 & 51.5501 & 48.4492 \\
3 & 53.5043 & 52.9523 & 50.5043 & 49.3936 & 48.3503 & 51.4403 & 49.3903 \\
4 & 53.9554 & 52.2902 & 51.9554 & 49.7863 & 48.7751 & 51.5855 & 48.3516 \\
5 & 53.6042 & 52.3925 & 50.6042 & 48.8776 & 48.0851 & 50.6551 & 48.5509 \\
6 & 54.2037 & 53.9670 & 49.2037 & 47.8695 & 47.1965 & 51.4965 & 48.5565 \\
7 & 53.4404 & 52.3465 & 50.4014 & 48.5321 & 47.6547 & 51.5547 & 49.5145 \\
8 & 54.6302 & 53.4509 & 49.6302 & 47.2283 & 46.7074 & 51.6475 & 48.6075 \\
9 & 53.6641 & 52.4761 & 50.6641 & 48.5963 & 47.7474 & 52.5364 & 49.5614 \\
10 & 52.2199 & 53.7228 & 51.2199 & 50.1466 & 48.8726 & 51.4827 & 48.5425 \\
\hline
\end{tabular}




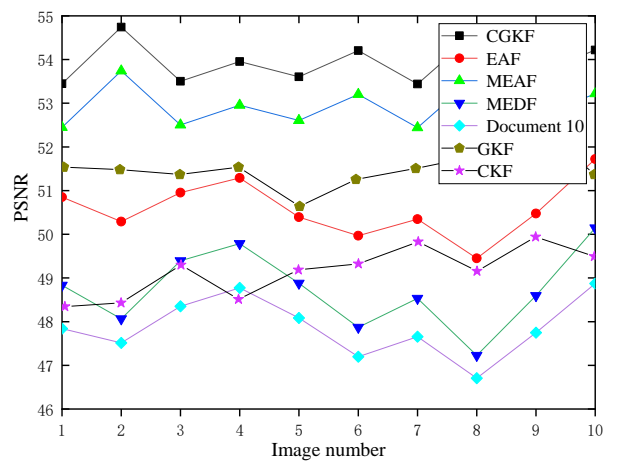

Figure 7. The psnr curves

\subsection{Image quality comparison after filtering}

\subsubsection{Filtered Images and Corresponding 3D Histogram}

The nlm filter with different kernel functions was used to denoise the weld images, and compare the filtering effect of various kernel functions (EKF, GKF, CKF and GCKF) as well as the algorithm in the literature.

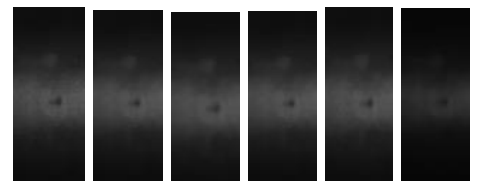

Figure 8 . The filtered images

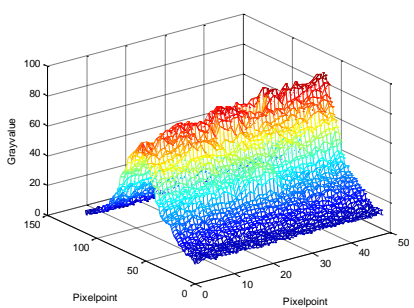

(a)

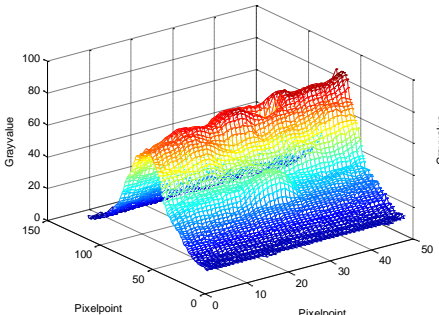

(d)

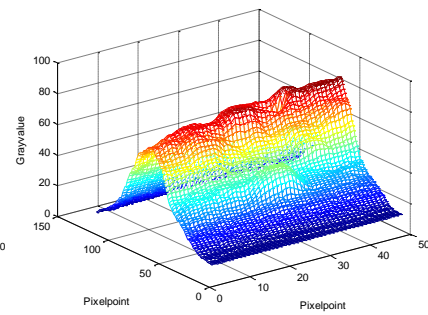

(b)

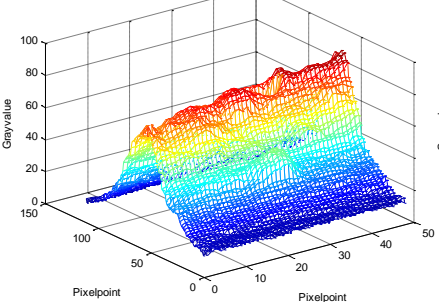

(e)

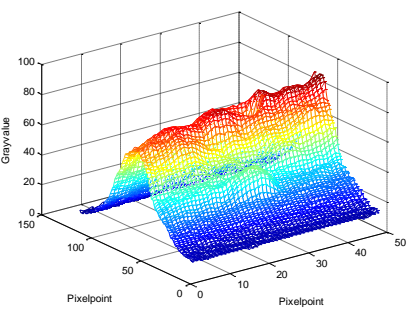

(c)

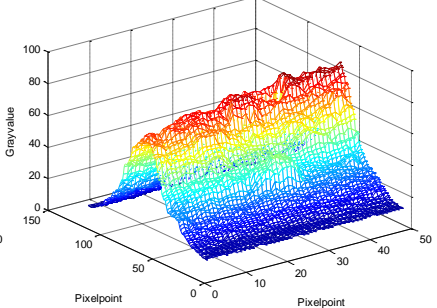

(f)

Figure 9. Three-dimensional histograms of filtered images

Figures 9(a) to (f) show the original image, filtered image (through cgkf NLM filter, mean filter, GKf NLM filter, EKF NLM filter, median filter, CKF NLM filter) filtered image and corresponding three-dimensional histogram. The simulation results show that the histogram of 3D image is smoother and the gray distribution is more uniform after cgkf NLM filtering, which indicates that cgkf NLM filter is most suitable for weld image filtering. 


\subsection{Enhanced image and corresponding histogram}

$\mathrm{XE}$ function, logarithmic function, histogram equalization and sin function were used to enhance the same randomly-selected weld images, and compare their enhancement effects with the algorithms in the literature.

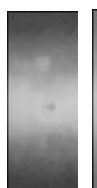

(a)

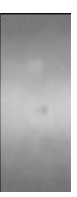

(b)

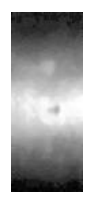

(c)

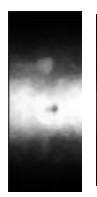

(d)

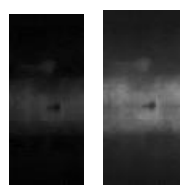

(e) (f)

Figure 10. Images enhanced by various methods

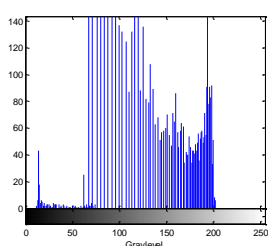

(a)

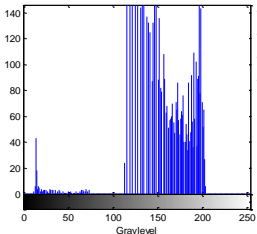

(b)

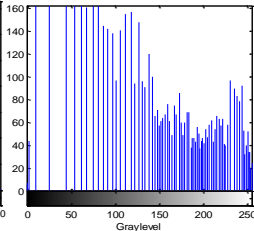

(c)

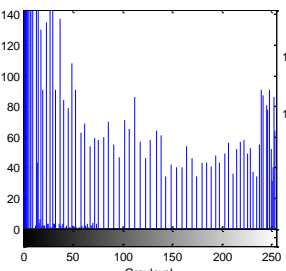

(d)

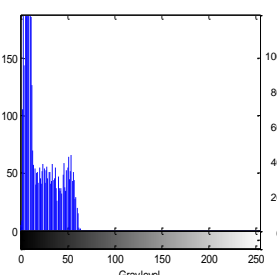

(e)

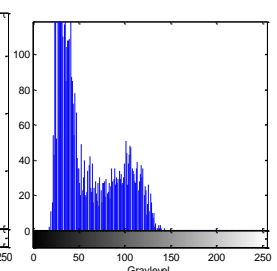

(f)

Figure 11. Histograms of enhanced images

Figure 10 (a)-(f) shows the images enhanced by xe function, logarithmic function, histogram equalization, sin function, and algorithms in Refs. 3 and 6. Figure 10 shows the gray histograms corresponding to Figure 11.

\section{Conclusions}

Through theoretical analysis and calculation, the mixed noise was separated in the image filtering part. For Gaussian noise, a new Gaussian cosine kernel nonlocal mean filtering algorithm was proposed by comparing the filtering effects of different kernel functions. Practical verification showed the new algorithm had obvious advantages over the traditional algorithm. Moreover, noise estimation was proposed for salt and pepper noise. This algorithm solved the problem of fixed median filter window and the inability to estimate noise intensity. Experiments showed the effect of noise reduction was obvious. In the image enhancement part, the ex function enhancement method proposed here can automatically and adaptively enhance the weld area instead of the background area. The best effect of human vision can be achieved by adjusting parameters without human intervention. Finally, the feasibility and advantages of the proposed method were verified by comparing with different algorithms. 


\section{ACKNOWLEDGEMENTS}

First of all, I would like to thank my tutor, Mr. Gao Weixin, for giving me a lot of guidance and suggestions in the process of writing the thesis; thirdly, I would like to thank Zhu Shiling for giving me a lot of opinions on the writing and grammar of English papers; finally, I would like to thank all the reviewers for their sincere opinions on the thesis, which benefited me a lot.

\section{REFERENCES}

[1] W. H.Wang, Image denoising algorithm based on noise detection and dynamic window, Journal of Graphics,40(1)(2019)112-115.

[2] Yahaghi E, Movafeghi A. Contrast enhancement of industrial radiography images by gabor filtering with automatic noise thresholding, Russian Journal of Nondestructive Testing, 55(1)(2019)73-79.

[3] Lin Z, Yingjie Z, Bochao D, Welding defect detection based on local image enhancement, Iet Image Processing,13(13)( 2019)2647-2658.

[4] Gharsallah M B, Mhammed I B, Braiek E B, Improved geometric anisotropic diffusion filter for radiography Image enhancement, Intelligent Automation and Soft Computing,24(2)(2018)231-240.

[5] Muthukumaran M, Prabaharan L, Sivapathi A,A comparative analysis of an anisitropic diffussion image denoising methods on weld x-radiography images,Far East Journal of Electronics and Communications, 17(2)(2017)267-281.

[6] Yahaghi E, Hosseiniashrafi M. Enhanced defect detection in radiography images of welded objects,Nondestructive Testing and Evaluation,34(1)(2019)13-22.

[7] Feng.Y, Chen.Z, Wang.D, Chen.J,et al. DeepWelding: a deep learning enhanced approach to gtaw using multi-source sensing images, IEEE Transactions on Industrial Informatics,1(16)(2020)465-474.

[8] W.L.Mu,G.J.Liu,Research on stochastic resonance enhancement of x-ray images based on a genetic algorithm,Non-Destructive Testing and Condition Monitoring,58(5)(2016)246-250.

[9] H.Liu.Research of non-local mean Image denoising algorithm based on structural similarity,master, Wuhan University of Technology,Wuhan,China,2017.

[10] $\mathrm{Su} \mathrm{H}$, Jung C. Enhancement of Low light images based on perceptual two-step noise suppression,IEEE Access,6(2018)7005-7018.

[11] N.H.Chang, The research of non-local means filtering to remove the image gaussian noise ,master,Xi an,University of Electronic Science and Technology of China,Chengdu,China,2015.

[12] Y.N.Liu,L.R.Wang,Research on fuzzy image enhancement based on laser sensor,Laser journal,41(3)(2020)123-125.

[13] H.Q.Zeng,Study on adaptive noise reduction of image sensor,Laser journal,12(67)(2019)67-71.

[14] C.Y.Zhu,X.Zhang,Research on laser 3D image enhancement system based on virtual reality,Laser journal,38(3)(2017)114-117.

[15] J.J.Liu,Design of edge adaptive enhancement device for low illumination nonlinear laser,Laser journal,38(3)(2017)122-125.

[16] D.D Chen,L. Chen.A new single image dehazing method based on improved double-area filter and guided filter,Journal of Computers,29(4)(2017),230-240.

[17] Khan S, Lee D H. An Adaptive Dynamically Weighted Median Filter for Impulse Noise Removal[J]. Eurasip Journal on Advances in Signal Processing, 2017, 2017(1):67.

[18] Balasubramanian G, Chilambuchelvan A,Vijayan S, etal. Probabilistic Decision Based Filter to Remove Impulse Noise Using Patch else Trimmed Median[J]. AEU-International Journal of Electronics and Communications, 2016, 70(4):471-481.

[19] Wang X T, Shen S S, Shi G M, et al. Iterative Non-Local Means Filter for Salt and Pepper Noise Removal[J]. Journal of Visual Communi- cation and Image Representation, 2016, 38(2016):440-450.

[20] Mohammad Tariqul Islam, SM Mahbubur Rahman, M Omair Ahmad, and MNS Swamy, "Mixed gaussian-impulse noise reduction from images using convolutional neural network," Signal Processing: Image Communication, vol. 68, pp. 26-41, 2018. 


\section{AUTHORS}

Zhang Xiangsong (1986 -), master degree, research direction: image processing.

Gao Weixin (1973 -), Professor, doctor, postgraduate supervisor, research direction: image and signal processing.

Zhu Shiling (1990 -), master degree, research direction: Internet of things technology and application.

(C) 2020 By AIRCC Publishing Corporation. This article is published under the Creative Commons Attribution (CC BY) license. 